\title{
Erratum and Addendum: New Approach to Einstein- Petrov Type I Spaces. II. Classification Scheme ${ }^{1}$
}

\author{
S. B. Edgar ${ }^{2}$
}

There is an error in the proof of Theorem 3(c); equation (35) does not follow from equation (32) as stated. However the result in Theorem 3(c) does hold, but for a smaller subclass of Class $\mathrm{C}$ spaces than claimed. A more appropriate subdivision of these spaces, with corrected statement and proof of Theorem 3, is given below.

The natural subdivision of Class $C$ spaces is:

(a) $A_{\mu}+k B_{,}-2 C_{\mu}=0$, where $k$ is a constant.

(b) $p A_{\mu}+q B_{\mu}+r C_{\mu}=0$, where $(p / r)$ and $(q / r)$ are analytic functions of $\Psi_{0}$ and $\Psi_{2}$.

(c) $p A_{\mu}+q B_{\mu}+r C_{\mu}=0$, where $(p / r)$ and $(q / r)$ are analytic functions of $\Psi_{0}, \Psi_{2}$, and $\Phi ; \Phi$ is a function of $\bar{\Psi}_{0}$ and $\bar{\Psi}_{2}$.

(d) Other than (a), (b), and (c).

These subclasses have the following properties:

Theorem 3.

(a) For Class C(a) spaces $A_{\mu}, B_{\mu}$, and $C_{\mu}$ are gradient vectors, i.e.,

$$
A_{\mu}=A_{, \mu}, \quad B_{\mu}=B_{, \mu}, \quad C_{\mu}=C_{, \mu}
$$

(b) For Class C(b) spaces $A_{\mu}, B_{\mu}$, and $C_{\mu}$ are hypersurface orthogonal vectors, i.e.,

$$
A_{\mu}=a A_{, \mu}, \quad B_{\mu}=b B_{, \mu}, \quad C_{\mu}=c C_{, \mu}
$$

${ }^{1}$ This paper appeared in International Journal of Theoretical Physics, 25, 425 (1986).

${ }^{2}$ Mathematics Department, University of Botswana, Gaborone, Botswana. 
(c) For Class C(c) spaces $A_{\mu}, B_{\mu}$, and $C_{\mu}$ have the form

$$
\begin{aligned}
& A_{\mu}=a A_{, \mu}+\alpha_{, \mu} \\
& B_{\mu}=b B_{, \mu}+\beta_{, \mu} \\
& C_{\mu}=c C_{, \mu}+\gamma_{, \mu}
\end{aligned}
$$

Proof of $(c)$. Substitution of ${ }^{3}$

$$
p A_{\mu}+q B_{\mu}+r C_{\mu}=0
$$

into (6) gives

$$
\begin{aligned}
& 3 \Psi_{2}\left\{r A_{[\mu ; \nu]}\right\}+\Psi_{0}\left\{r B_{[\mu ; \nu]}+2(2 p+r) A_{[\mu} B_{\nu]}\right\}=0 \\
& 3 \Psi_{2}\left\{r B_{[\mu ; \nu]}-2(2 p+r) A_{[\mu} B_{\nu]}\right\} \\
& \quad+\Psi_{0}\left\{(r-4 p) A_{[\mu ; \nu]}-4 q B_{[\mu ; \nu]}-4 r(p / r)_{,[\nu} A_{\mu]}\right. \\
& \left.\quad-4 r(q / r)_{[\nu} B_{\mu]}\right\}=0
\end{aligned}
$$

Since $(p / r)$ and $(q / r)$ are functions of $\Psi_{0}, \Psi_{2}$, and $\Phi\left(\bar{\Psi}_{0}, \bar{\Psi}_{2}\right)$, equation (25) has the form, after using equations (5),

$$
\begin{aligned}
& 3 \Psi_{2}\left\{r B_{[\mu ; \nu]}-2(2 p+r) A_{[\mu} B_{\nu]}\right\} \\
& \quad+\Psi_{0}\left\{(r-4 p) A_{[\mu ; \nu]}-4 q B_{[\mu ; \nu]}+r f A_{[\mu} B_{\nu]}\right. \\
& \left.\quad+r g A_{[\mu} \Phi_{, \nu]}+r h B_{[\mu} \Phi_{, \nu]}\right\}=0
\end{aligned}
$$

where $f, g$, and $h$ are functions of $\Psi_{0}, \Psi_{2}$, and $\Phi\left(\bar{\Psi}_{0}, \bar{\Psi}_{2}\right)$.

With the choice of $G_{\mu \nu}$ as

$$
G_{\mu \nu}=r f A_{[\mu} B_{\nu]}+r g A_{[\mu} \Phi_{, \nu]}+r h B_{[\mu} \Phi_{, \nu]}
$$

and noting

$$
\begin{aligned}
G_{[\mu \nu} A_{\lambda} B_{\rho]} & =0 \\
G_{[\mu \nu} G_{\rho \lambda]} & =0
\end{aligned}
$$

the rest of the proof, from equation (29) to the end, is now valid, for the redefined class $\mathrm{C}(\mathrm{c})$ spaces.

Finally, it is noted that there is no obvious simplification in the form of the vectors for the subclass $\mathrm{C}(\mathrm{d})$ spaces.

${ }^{3}$ Equation numbers refer to original paper. 